\title{
Modeling of three-dimensional cutting forces in micro-end-milling
}

\author{
Chengfeng $\mathrm{Li}^{1}$, Xinmin $\mathrm{Lai}^{1}$, Hongtao $\mathrm{Li}^{1}$ and ${\mathrm{Jun} \mathrm{Ni}^{2}}^{2}$ \\ ${ }^{1}$ School of Mechanical and Dynamic Engineering, Shanghai Jiao Tong University, Shanghai, \\ 200240, People's Republic of China \\ ${ }^{2}$ Department of Mechanical Engineering and Applied Mechanics, University of Michigan, \\ Ann Arbor, MI 48109, USA \\ E-mail: abellcf@sjtu.edu.cn
}

Received 4 October 2006, in final form 3 January 2007

Published 22 February 2007

Online at stacks.iop.org/JMM/17/671

\begin{abstract}
A new nominal uncut chip thickness algorithm for micro-scale end-milling is proposed by considering the combination of an exact trochoidal trajectory of the tool tip and tool run-out, and then the actual uncut chip thickness may be obtained from a comparison between the current accumulative uncut chip thickness and the minimum chip thickness. Due to the intermittency of the chip formation, the milling process is divided into an elastic-plastic deformation regime and a chip formation regime dominated by ploughing forces and shearing forces, respectively, and three-dimensional cutting forces are modeled according to different regimes. Based on the modeling and simulation technologies introduced, a simulation system for the prediction of three-dimensional cutting forces of a micro-scale end-milling process is developed. The simulation results show a very satisfactory agreement with those data from milling experiments.
\end{abstract}

(Some figures in this article are in colour only in the electronic version)

\section{Introduction}

The development of miniaturized technologies has become a global phenomenon that continues to make marked impact across a broad application domain. Miniature components are needed for a wide range of applications such as avionics, biotechnology, communications, electronics, medicine, optics and so on. Miniaturization of many consumer products and esthetic goals drastically increased micro-end-milling operation applications in the conventional shop floor.

In most micro-end-milling operations, the diameters of a micro cutting tool vary from $0.1 \mathrm{~mm}$ to $1 \mathrm{~mm}$, and stress variation on the tiny shaft of the micro cutting tool is much higher than that on a conventional scale tool, which drastically shortens the tool's life. If the cutting conditions are not selected properly, micro-tools will be broken in a few seconds. So a precise estimation of the cutting forces of micro-end-milling plays an important role in guiding the selection of cutting conditions in order to economically obtain high machining quality and ensure as long a tool life as possible.

Tlusty and MacNeil (1975) developed closed-form expressions for the milling force, assuming a circular tool path and a constant proportionality between the cutting force and chip load. Other researchers modeled the end-milling process by discretizing the tool into axial slices and considering both circular and trochoidal tool paths (Kline et al 1982, Montgomery and Altintas 1991).

However, conventional milling process models have been applied to micro-milling with only limited success. At the micro-scale, there are phenomenological differences in the milling process that cannot be described by the simple scaling of the conventional scale milling process. The critical differences at the micro-scale arise from the breakdown of the assumptions of negligible edge radius effects. Weule et al (2001) determined that the roundness of a cutting edge is more significant in micro-scale milling. As the size of a tool decreases, the sharpness of the tool cannot be improved proportionally due to limitations in the tool fabrication processes and reduction in the structural strength of the tool. As a result, the feed per tooth in micro-milling may be comparable to or even less than the cutting edge radius because of the required range of process parameters for a stable machining scale with the process. It has been found that there 
exists a minimum chip thickness below which no chip will form (Yuan et al 1996). Kim et al (2004) experimentally determined that when the feed per tooth is comparable with the edge radius of the tool, as is often the case in micro-milling processes, the chip formation process becomes intermittent and the conventional understanding that a chip is formed with every tooth pass is no longer valid. According to their model, the minimum chip thickness of various combinations of tools and workpiece materials may be estimated based on easily attainable cutting force data.

On the other hand, feed per tooth to tool radius ratio is large enough so that it is not negligible in micro-end-milling; therefore the trajectory of the tool tip cannot be simplified as a circle. Furthermore, a small run-out that affects the cutting force profile of conventional end-milling operations creates very little drastic force variations in micro-end-milling operations, and a relatively large tool run-out will result in the feed per tooth for each tooth pass to be no longer a constant under a given cutting condition.

To calculate the cutting forces of micro-end-milling, many modified models had been founded. Volger et al (2003, 2004) incorporated the minimum chip thickness concept for predicting the effects of the cutter edge radius on the cutting forces. Bao and Tansel (2000a) proposed a new analytical cutting force model which calculated the chip thickness by considering the trajectory of the tool tip while the tool rotated and moved ahead continuously. The effect of run-out was noted in their later research (Bao and Tansel 2000b, 2000c). In their model, it is observed that the model gives a good result at a higher feed rate. But since the diameter of the milling tools used in their experiments seems relatively large, they did not consider the effect of the intermittency of the chip formation observed at low feeds per tooth. Zaman et al (2006) proposed a three-dimensional analytical cutting force model for the micro-end-milling operation. Their model determines the theoretical chip area at any specific angular position of the tool cutting edge by considering the geometry of the path of the cutting edge, and relates this with the tangential cutting force.

This study develops a prediction model of a threedimensional cutting force for micro-end-milling operation that is able to describe the intermittency of the chip formation process. Section 2 proposes a new nominal uncut chip thickness algorithm by considering the combination of the exact trochoidal trajectory of the tool tip and tool run-out, and a relationship describing the resultant periodicity in the actual uncut chip thickness as a function of the nominal uncut chip thickness at the angle of interest and the value of minimum chip thickness. Three-dimensional cutting forces, modeled according to an elastic-plastic deformation regime and a shearing-ploughing regime, are given in section 3. A series of experimental investigations were undertaken to verify the model by comparing the simulated cutting forces with the experimental data. The experimental setup and the result analyses are presented in section 4 .

\section{Instantaneous uncut chip thickness algorithm}

The conventional uncut chip thickness can be expressed by the following equation based on the assumption of a circular tool

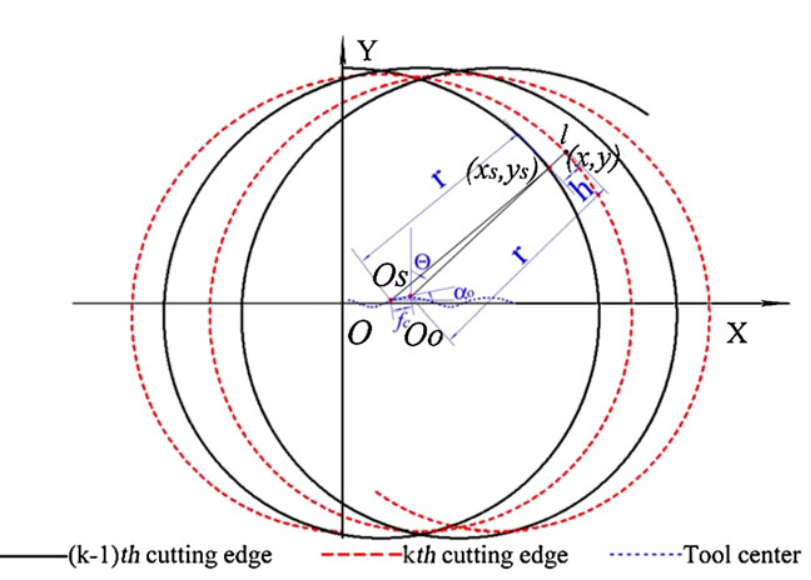

Figure 1. Coordinate system of micro-end-milling operations.

path:

$$
h_{\mathrm{n}}(\theta)=f_{\mathrm{t}} \sin \theta,
$$

where $h_{\mathrm{n}}$ represents nominal uncut chip thickness (mm) for a position angle $\theta(\mathrm{rad})$ and $f_{\mathrm{t}}$ is the feed per tooth $(\mathrm{mm} /$ tooth).

However, as the end-milling process is scaled down at the micro-scale, high speed spindles with more than $30000 \mathrm{rpm}$ are usually utilized to supply enough cutting speed. Their run-out values vary from $1 \mu \mathrm{m}$ to $5 \mu \mathrm{m}$, and feeds per tooth range from $0.1 \mu \mathrm{m}$ to $10 \mu \mathrm{m}$; thus the tool run-out to tool diameter ratio becomes very large compared to the conventional scale end-milling, and creates drastic variations of uncut chip thickness. In addition, the intermittency of the chip formation process dominates most of micro-scale endmilling operations. Thus, the conventional computation model of uncut chip thickness cannot precisely describe the actual chip formation of micro-end-milling.

\subsection{A new nominal uncut chip thickness algorithm}

The trajectory of the tool tip in end-milling operations is illustrated in figure 1 where a real line curve and dashed line curve represent the trajectories of tool tips of $(k-1)$ th and $k$ th cutting edges, respectively, where $k=0,1,2, \ldots, K-1$ and $K$ is the number of tool teeth. The zero angle is defined as a position where the $(k-1)$ th cutting edge is aligned with $y$-axial (cross-feed direction).

Considering the effect of the tool run-out, the trajectory of the tool center can be written as

$$
\begin{aligned}
& x=r_{\mathrm{o}} \sin (\omega t+\gamma)+f t / 60, \\
& y=r_{\mathrm{o}} \cos (\omega t+\gamma),
\end{aligned}
$$

where $f$ is the feed rate $\left(\mathrm{mm} \mathrm{min}^{-1}\right), \omega$ is the spindle circle speed $\left(\mathrm{rad} \mathrm{s}^{-1}\right), r_{\mathrm{o}}$ is the run-out length $(\mathrm{mm})$ and $\gamma$ is the run-out angle (rad). The trajectory of the tool center with the tool run-out is represented by the dotted line curve in figure 1 .

So the trajectory of the $k$ th tool tip may be written as

$$
\begin{aligned}
& x(t, k)=f t / 60+r \sin (\omega t-2 \pi k / K)+r_{\mathrm{o}} \sin (\omega t+\gamma) \\
& y(t, k)=r \cos (\omega t-2 \pi k / K)+r_{\mathrm{o}} \cos (\omega t+\gamma),
\end{aligned}
$$

where $r$ is the tool radius ( $\mathrm{mm})$.

The coordinate of the $k$ th tool tip at time $t$ with the position angle $\theta$ is $(x, y)$, the line connecting point $(x, y)$ and its 
corresponding tool center $O_{0}$ is denoted as line $l$ and the point $\left(x_{\mathrm{s}}, y_{\mathrm{s}}\right)$, the intersection between the line $l$ and the trajectory of the $(k-1)$ th cutting edge, means the position of the $(k-1)$ th cutting edge at time $t_{\mathrm{s}}$. The line $l$ can be expressed by the following equation:

$$
\begin{aligned}
y= & \tan ^{-1}(\omega t-2 \pi k / K)[x-f t / 60 \\
& \left.-r_{\mathrm{o}} \sin (\omega t+\gamma)\right]+r_{\mathrm{o}} \cos (\omega t+\gamma) .
\end{aligned}
$$

Furthermore, the trajectory of the $(k-1)$ th flute can be deduced from equation (3) by replacing $k$ with $(k-1)$ and $t$ with $t_{\mathrm{s}}$. Using the condition that the point $\left(x_{\mathrm{s}}, y_{\mathrm{s}}\right)$ is on the line $l$, the time $t_{\mathrm{s}}$ can be solved from the following equation:

$$
\begin{aligned}
& r \tan (\omega t-2 \pi k / K) \cos \left[\omega t_{\mathrm{s}}-2 \pi(k-1) / K\right] \\
& \quad+r_{\mathrm{o}} \tan (\omega t-2 \pi k / K) \cos \left(\omega t_{\mathrm{s}}+\gamma\right) \\
& \quad-r_{\mathrm{o}} \tan (\omega t-2 \pi k / K) \cos (\omega t+\gamma) \\
& \quad-f t_{\mathrm{s}} / 60+f t / 60-r \sin \left[\omega t_{\mathrm{s}}-2 \pi(k-1) / K\right] \\
& \quad-r_{\mathrm{o}} \sin \left(\omega t_{\mathrm{s}}+\gamma\right)+r_{\mathrm{o}} \sin (\omega t+\gamma)=0 .
\end{aligned}
$$

The distance between the two tool center points $O_{\mathrm{s}}$ and point $O_{0}$ which correspond to time $t_{\mathrm{s}}$ and time $t$ respectively, namely $f_{\mathrm{c}}$, can be calculated by

$$
f_{\mathrm{c}}=\left[\left(x_{\mathrm{o}_{\mathrm{o}}}-x_{\mathrm{o}_{\mathrm{s}}}\right)^{2}+\left(y_{\mathrm{o}_{\mathrm{o}}}-y_{\mathrm{o}_{\mathrm{s}}}\right)^{2}\right]^{0.5} \text {. }
$$

From the geometric relationship, it leads to

$$
\begin{aligned}
& h_{\mathrm{n}}(t, k)=r+f_{\mathrm{c}} \sin \left(\omega t-2 \pi k / K+\alpha_{\mathrm{o}}\right) \\
& -\left[r^{2}-f_{\mathrm{c}}^{2} \cos ^{2}\left(\omega t-2 \pi k / K+\alpha_{\mathrm{o}}\right)\right]^{0.5},
\end{aligned}
$$

where

$$
\alpha_{\mathrm{o}}=\arctan \left(\frac{y_{\mathrm{o}_{\mathrm{o}}}-y_{\mathrm{o}_{\mathrm{s}}}}{x_{\mathrm{o}_{\mathrm{o}}}-x_{\mathrm{o}_{\mathrm{s}}}}\right) .
$$

The proposed model indicates that one obstacle to the calculation of nominal chip thickness is the solution of time $t_{\mathrm{s}}$ from equation (5) since it is a complex nonlinear polynomial equation. As the Newton-Raphson iterative method is characterized by quadratic convergence, the proposed model utilizes it to solve time $t_{\mathrm{s}}$. Once the value of $t_{\mathrm{s}}$ is determined, the nominal uncut chip thickness $h_{\mathrm{n}}$ will be easily obtained by solving equations (6)-(8).

For the Newton-Raphson iterative method, it is essential to select a proper initial iterative value. Considering the periodicity of chip formation, the following initial value of $t_{\mathrm{s}}$ is used:

$$
t_{\mathrm{s}_{0}}=t-2 \pi /(\omega Z) .
$$

Suppose that $F\left(t_{\mathrm{s}}\right)$ is a function of $t_{\mathrm{s}}$, namely equal to the left of equation (5), according to the Newton-Raphson iterative method for each iterative loop, $t_{\mathrm{s}_{i}}$ can be updated according to the value of $t_{\mathrm{s}_{i+1}}$ which can be determined from the following equation:

$$
t_{\mathrm{s}_{i+1}}=t_{\mathrm{s}_{i}}-\frac{F\left(t_{\mathrm{s}_{i}}\right)}{F^{\prime}\left(t_{\mathrm{s}_{i}}\right)}
$$

where $i=0,1,2, \ldots$.

The procedure for solving the nominal chip thickness $h_{\mathrm{n}}$ corresponding to a specified value of time $t$ is shown in figure 2. It is found that the value of $t_{\mathrm{s}}$ corresponding to any value of time $t$ will converge only through less than five iterative loops even though $\varepsilon$ is as small as $0.001 \%$ of tool radius.

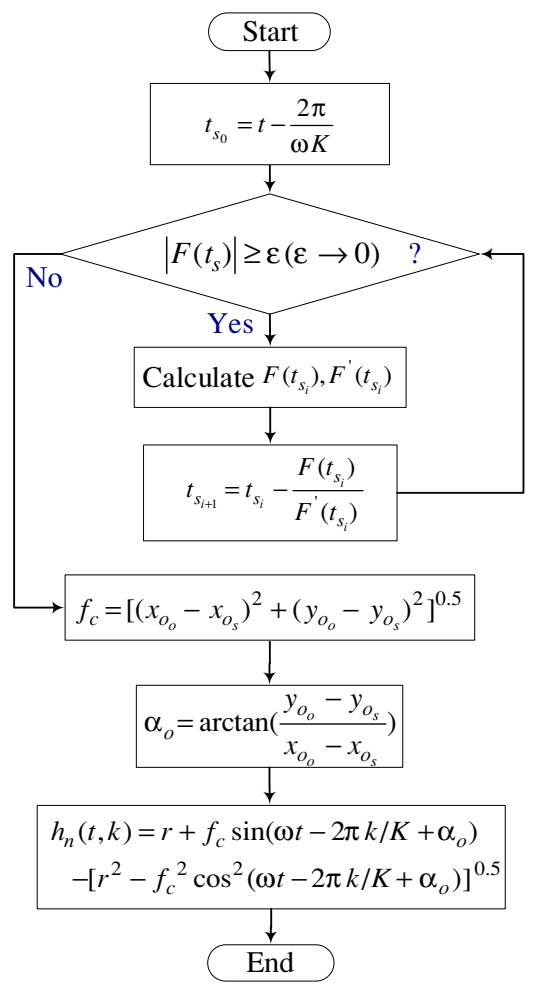

Figure 2. Procedure of the iterative algorithm for the nominal uncut chip thickness of micro-end-milling.

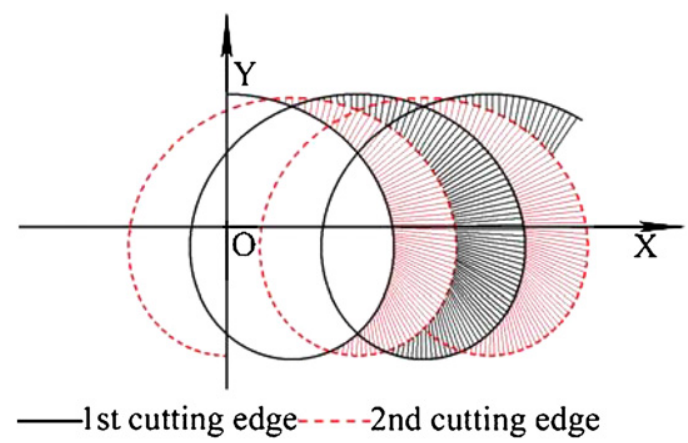

Figure 3. Graph of determined chip thickness by the proposed algorithm for a two-flute tool with a $45^{\circ}$ tool run-out angle.

In figure 3, the real line curve and the dashed line curve represent the milling trajectories of the first and second cutting edges, respectively, for a two-flute milling tool. The length of each of the black and gray (or red in multicolor print) real lines, determined by the method proposed in this paper, represents the uncut chip thickness of the first and second cutting edges, respectively, when the corresponding edge arrives at the specified point on the trajectory. The areas hatched by black real lines and gray (or red in multicolor print) real lines represent the areas which are cut by the first and second cutting edges, respectively. It is distinct that the real lines occupy the area between the real line curve and the dashed line curve exactly. That means the nominal uncut chip thickness algorithm proposed can accurately describe the process of milling.

Furthermore, it is found that under a given cutting condition, the chip thickness values corresponding to the 


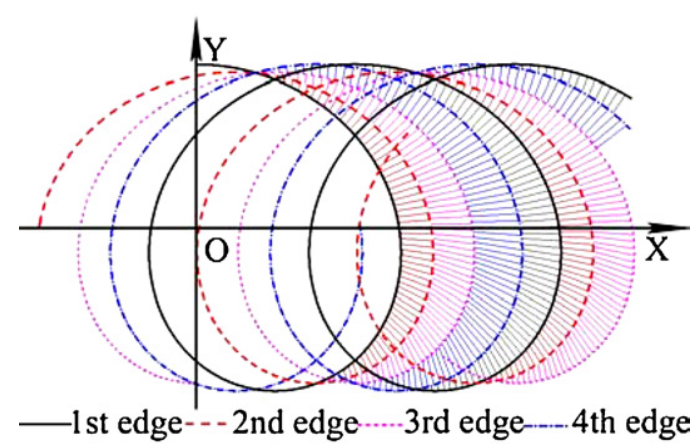

Figure 4. Graph of determined chip thickness by the proposed algorithm for a four-flute tool with a $45^{\circ}$ tool run-out angle.

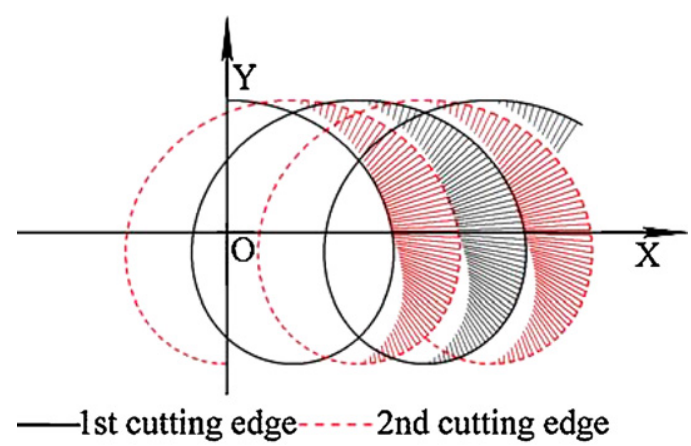

Figure 5. Graph of determined chip thickness by a conventional algorithm for a two-flute tool.

first and second cutting edges at the same position angle are unequal while they are equal without the tool run-out. This is because the trajectories of the two cutting edges are distinctly influenced by the tool run-out, which results in the feed per tooth for different tooth passes to be no longer a constant. It is also found through varying tool run-out lengths that the variation of feeds per tooth for the tooth passes proportionately increases with the tool run-out length. Even with the strong influence of tool run-out, the proposed algorithm can still obtain accurately nominal uncut chip thickness values at any time. Also, the state of micro-end-milling operations with a four-flute milling tool is shown in figure 4 and the same conclusions can be obtained.

In comparison, figure 5 shows the graph of the nominal chip thickness value for a two-flute tool determined by the conventional algorithm. It is obvious that during the initial and final phases of the cutting edge engaging with the workpiece, areas taken up by black and gray (or red in multicolor print) real lines do not occupy the whole areas between the trajectories of the first and second cutting edges. This means that the chip thickness values determined by the conventional algorithm cannot represent the practical state correctly. The error of the algorithm is due to the negligence of an exact trochoidal trajectory of the tool tip and tool run-out in chip formation modeling, but such an error is unbearable for micro-scale endmilling.

\subsection{Actual uncut chip thickness algorithm}

The chip formation in micro-scale milling may be inconsistent and result in several noncutting tooth passes, particularly at low feed per tooth. When the current engagement $h_{\mathrm{a}}(t, k)$ is smaller than the minimum chip thickness, the workpiece elastically and plastically deforms under the interaction with the milling cutter, no material is assumed to be removed from the workpiece and the current value of uncut chip thickness is added to that of the next tooth pass at the same position angle. So the actual uncut chip thickness of the next tooth pass may be expressed as

$h_{\mathrm{a}}\left(t+\frac{2 \pi}{\omega K}, k+1\right)=h_{\mathrm{a}}(t, k)+h_{\mathrm{n}}\left(t+\frac{2 \pi}{\omega K}, k+1\right)$,

when $h_{\mathrm{a}}(t, k)<h_{\min }$, where $h_{\min }$ is the minimum chip thickness.

In contrast, when the current accumulative engagement $h_{\mathrm{a}}(t, k)$ is larger than the minimum chip thickness, the interaction forces of the workpiece with the cutter are dominated by shearing forces, and all material as thick as the actual engagement is assumed to be removed as a chip. So the actual uncut chip thickness of the next tooth pass can be determined by the following equation:

$$
h_{\mathrm{a}}\left(t+\frac{2 \pi}{\omega K}, k+1\right)=h_{\mathrm{n}}\left(t+\frac{2 \pi}{\omega K}, k+1\right)
$$

when $h_{\mathrm{a}}(t, k) \geqslant h_{\min }$.

\section{Cutting force modeling of the micro end-milling operation}

\subsection{Calculation of entry angle and exit angle}

To calculate the cutting forces by using the cutting force model, four computational parameters are introduced.

The workpiece cutting angle $\varphi(\mathrm{rad})$ is defined as

$$
\varphi=\arccos \left(\frac{r-d_{\mathrm{r}}}{r}\right)
$$

where $d_{\mathrm{r}}$ is the radial depth of the cut ( $\left.\mathrm{mm}\right)$.

The tool cutter angle $\psi(\mathrm{rad})$ is defined as

$$
\psi=2 \pi / K
$$

The engagement angle $\alpha(\mathrm{rad})$ is defined as

$$
\alpha=d_{\mathrm{a}} \tan \beta / r
$$

where $d_{\mathrm{a}}$ is the axial depth of the cut (mm) and $\beta$ is the helix angle of the cutter (rad).

The position angle $\theta(t, k)(\mathrm{rad})$ of the $k$ th tooth at time $t$ is defined as

$$
\theta(t, k)=\omega t-\frac{2 \pi k}{K}
$$

Under the specified cutting conditions that the engagement angle $\alpha$ is less or more than the workpiece cutting angle $\varphi$ and the milling method is up-milling or down-milling, the entry angle $\theta_{\mathrm{en}}$ and exit angle $\theta_{\mathrm{ex}}$ at different sections of the position angle $\theta(t, k)$ can be obtained from table 1 . 
Table 1. Calculation of entry angle and exit angle.

\begin{tabular}{|c|c|c|c|c|c|c|}
\hline & \multicolumn{3}{|c|}{ Up-milling } & \multicolumn{3}{|c|}{ Down-milling } \\
\hline & $\bar{\theta}$ & $\theta_{\text {en }}$ & $\overline{\theta_{\mathrm{ex}}}$ & $\bar{\theta}$ & $\theta_{\mathrm{en}}$ & $\overline{\theta_{\mathrm{ex}}}$ \\
\hline \multirow{3}{*}{$\alpha \leqslant \varphi$} & $0 \leqslant \theta<\alpha$ & 0 & $\theta$ & $\pi-\varphi \leqslant \theta<\pi-\varphi+\alpha$ & $\pi-\varphi$ & $\theta$ \\
\hline & $\alpha \leqslant \theta<\varphi$ & $\theta-\alpha$ & $\theta$ & $\pi-\varphi+\alpha \leqslant \theta<\pi$ & $\theta-\alpha$ & $\theta$ \\
\hline & $\varphi \leqslant \theta \leqslant \varphi+\alpha$ & $\theta-\alpha$ & $\varphi$ & $\pi \leqslant \theta \leqslant \pi+\alpha$ & $\theta-\alpha$ & $\pi$ \\
\hline \multirow{3}{*}{$\alpha>\varphi$} & $0 \leqslant \theta<\varphi$ & 0 & $\theta$ & $\pi-\varphi \leqslant \theta<\pi$ & $\pi-\varphi$ & $\theta$ \\
\hline & $\varphi \leqslant \theta<\alpha$ & 0 & $\varphi$ & $\pi \leqslant \theta<\pi-\varphi+\alpha$ & $\pi-\varphi$ & $\pi$ \\
\hline & $\alpha \leqslant \theta \leqslant \varphi+\alpha$ & $\theta-\alpha$ & $\varphi$ & $\pi-\varphi+\alpha \leqslant \theta \leqslant \pi+\alpha$ & $\theta-\alpha$ & $\pi$ \\
\hline
\end{tabular}

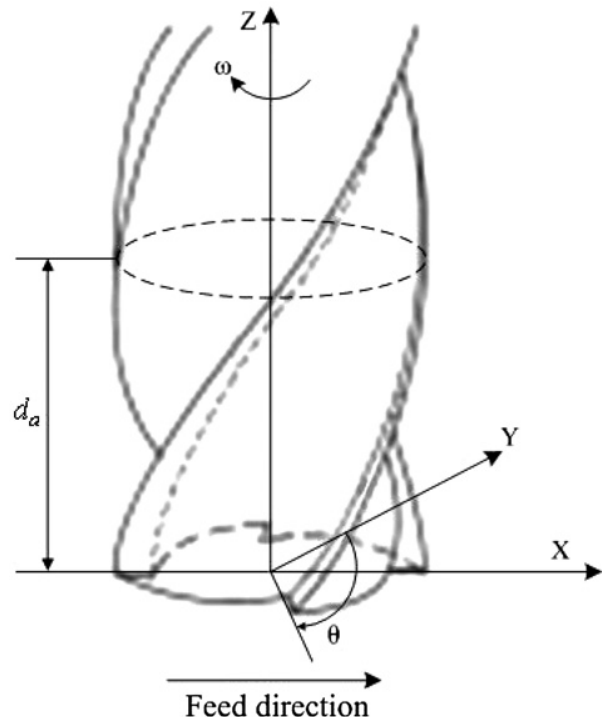

Figure 6. Model coordinate system of end-milling operations.

\subsection{Three-dimensional cutting force model}

The model coordinate system of end-milling is shown in figure 6 . This section will respectively model cutting forces according to two different milling regimes.

(i) When the actual uncut chip thickness $h_{\mathrm{a}}(t, k)$ is smaller than the minimum chip thickness, the workpiece elastically and plastically deforms under the interaction with the milling cutter, and no material is assumed to be removed from the workpiece. As a way of describing the relationships of forces and actual engagement, the tangential force $F_{\mathrm{t}}$, radial force $F_{\mathrm{r}}$ and axial force $F_{\mathrm{a}}$ are modeled as power functions (Kim et al 2004) described by

$$
\begin{aligned}
& \mathrm{d} F_{\mathrm{t}}(t, k, z)=A_{\mathrm{t}} h_{\mathrm{a}}(t, k, z)^{B_{\mathrm{t}}} \mathrm{d} z \\
& \mathrm{~d} F_{\mathrm{r}}(t, k, z)=A_{\mathrm{r}} h_{\mathrm{a}}(t, k, z)^{B_{\mathrm{r}}} \mathrm{d} z \\
& \mathrm{~d} F_{\mathrm{a}}(t, k, z)=A_{\mathrm{a}} h_{\mathrm{a}}(t, k, z)^{B_{\mathrm{a}}} \mathrm{d} z,
\end{aligned}
$$

where $A_{\mathrm{t}}$ and $B_{\mathrm{t}}$ are tangential force coefficients, $A_{\mathrm{r}}$ and $B_{\mathrm{r}}$ are radial force coefficients and $A_{\mathrm{a}}$ and $B_{\mathrm{a}}$ are axial force coefficients. These six coefficients are constant for the specified cutter and workpiece material, and could be acquired by curve fitting the measured force data into the force model using the least-squares method.

(ii) When the actual uncut chip thickness $h_{\mathrm{a}}(t, k)$ is larger than the minimum chip thickness, the interaction forces of the workpiece with the cutter are dominated by shearing forces, and all material as thick as the actual engagement is assumed to be removed as a chip. The tangential cutting force $F_{\mathrm{t}}$, radial cutting force $F_{\mathrm{r}}$ and axial cutting force $F_{\mathrm{a}}$ are modeled as (Wang et al 2002)

$$
\begin{aligned}
& \mathrm{d} F_{\mathrm{t}}=A_{\mathrm{ts}} h_{\mathrm{a}}(t, k, z) \mathrm{d} z+B_{\mathrm{tp}} \mathrm{d} z \\
& \mathrm{~d} F_{\mathrm{r}}=A_{\mathrm{rs}} h_{\mathrm{a}}(t, k, z) \mathrm{d} z+B_{\mathrm{rp}} \mathrm{d} z \\
& \mathrm{~d} F_{\mathrm{a}}=A_{\text {as }} h_{\mathrm{a}}(t, k, z) \mathrm{d} z+B_{\mathrm{ap}} \mathrm{d} z,
\end{aligned}
$$

where $A_{\mathrm{ts}}$ and $B_{\mathrm{tp}}$ are tangential shearing and ploughing force coefficients; $A_{\mathrm{rs}}$ and $B_{\mathrm{rp}}$ are radial shearing and ploughing force coefficients and $A_{\text {as }}$ and $B_{\text {ap }}$ are axial shearing and ploughing force coefficients, respectively. These six coefficients can also be acquired by curve fitting the measured force data into the force model using the least-squares method.

The three-dimensional cutting forces, $F_{x}, F_{y}$ and $F_{z}$, can be expressed as

$$
\left[\begin{array}{l}
\mathrm{d} F_{x} \\
\mathrm{~d} F_{y} \\
\mathrm{~d} F_{z}
\end{array}\right]=\left[\begin{array}{ccc}
-\cos \theta & -\sin \theta & 0 \\
\sin \theta & -\cos \theta & 0 \\
0 & 0 & 1
\end{array}\right]\left[\begin{array}{l}
\mathrm{d} F_{\mathrm{t}} \\
\mathrm{d} F_{\mathrm{r}} \\
\mathrm{d} F_{\mathrm{a}}
\end{array}\right] .
$$

Considering the geometric conditions,

$$
\frac{r \mathrm{~d} \theta}{\mathrm{d} z}=\tan \beta
$$

and taking the integration, the cutting force expressions are

$$
\begin{gathered}
F_{x}(t)=\frac{r}{\tan \beta} \sum_{k=0}^{K-1} \int_{\theta_{\mathrm{en}}}^{\theta_{\mathrm{ex}}}\left[-A_{\mathrm{t}} h_{\mathrm{a}}(\theta, k)^{B_{\mathrm{t}}} \cos \theta\right. \\
\left.-A_{\mathrm{r}} h_{\mathrm{a}}(\theta, k)^{B_{\mathrm{r}}} \sin \theta\right] \mathrm{d} \theta \\
F_{y}(t)=\frac{r}{\tan \beta} \sum_{k=0}^{K-1} \int_{\theta_{\mathrm{en}}}^{\theta_{\mathrm{ex}}}\left[A_{\mathrm{t}} h_{\mathrm{a}}(\theta, k)^{B_{\mathrm{t}}} \sin \theta\right. \\
\left.-A_{\mathrm{r}} h_{\mathrm{a}}(\theta, k)^{B_{\mathrm{r}}} \cos \theta\right] \mathrm{d} \theta \\
F_{z}(t)=\frac{r}{\tan \beta} \sum_{k=0}^{K-1} \int_{\theta_{\mathrm{en}}}^{\theta_{\mathrm{ex}}} A_{\mathrm{a}} h_{\mathrm{a}}(\theta, k)^{B_{\mathrm{a}}} \mathrm{d} \theta
\end{gathered}
$$

when $h_{\mathrm{a}}(\theta, k)<h_{\text {min }}$

$$
\begin{gathered}
F_{x}(t)=\frac{r}{\tan \beta} \sum_{k=0}^{K-1} \int_{\theta_{\mathrm{en}}}^{\theta_{\mathrm{ex}}}\left\{-\left[A_{\mathrm{ts}} h_{\mathrm{a}}(\theta, k)+B_{\mathrm{tp}}\right] \cos \theta\right. \\
\left.-\left[A_{\mathrm{rs}} h_{\mathrm{a}}(\theta, k)+B_{\mathrm{rp}}\right] \sin \theta\right\} \mathrm{d} \theta \\
F_{y}(t)=\frac{r}{\tan \beta} \sum_{k=0}^{K-1} \int_{\theta_{\mathrm{en}}}^{\theta_{\mathrm{ex}}}\left\{\left[A_{\mathrm{ts}} h_{\mathrm{a}}(\theta, k)+B_{\mathrm{tp}}\right] \sin \theta\right. \\
\left.-\left[A_{\mathrm{rs}} h_{\mathrm{a}}(\theta, k)+B_{\mathrm{rp}}\right] \cos \theta\right\} \mathrm{d} \theta \\
F_{z}(t)=\frac{r}{\tan \beta} \sum_{k=0}^{K-1} \int_{\theta_{\mathrm{en}}}^{\theta_{\mathrm{ex}}}\left[A_{\mathrm{as}} h_{\mathrm{a}}(\theta, k)+B_{\mathrm{ap}}\right] \mathrm{d} \theta
\end{gathered}
$$

when $h_{\mathrm{a}}(\theta, k) \geqslant h_{\min }$. 


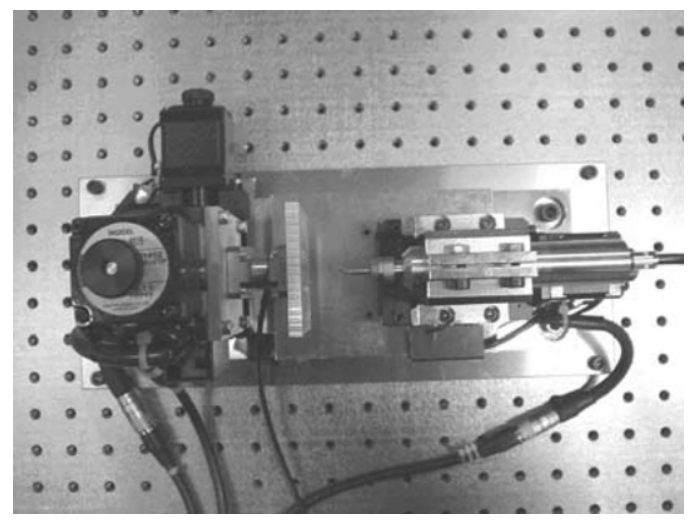

Figure 7. The developed micro-milling machine.

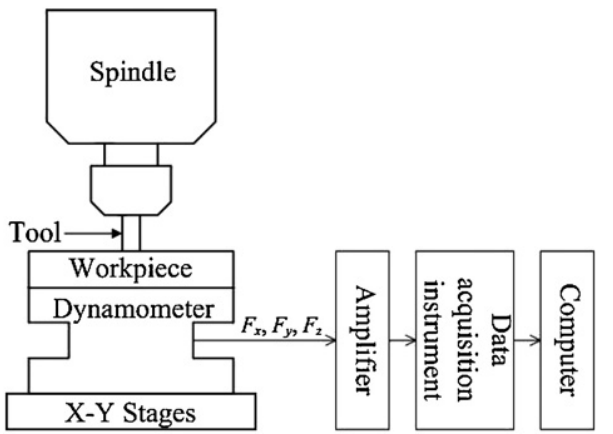

Figure 8. Experimental setup.

\section{Model verification}

\subsection{Experimental setup}

More than 200 cutting experiments of micro-scale end-milling operations were performed on a developed micro-milling machine, as shown in figure 7. The feed system of this machine consists of three precision linear stages with a high feed resolution, i.e. $50 \mathrm{~nm}$, and each stage is driven by the stepping motor. An air motor spindle with a maximum rotating speed of $120000 \mathrm{rpm}$ provides enough high speed to ensure cutting velocity. The diameters of the used two-flute micromilling tools vary from $0.1 \mathrm{~mm}$ to $1 \mathrm{~mm}$.

A typical experimental setup is presented in figure 8 . A Kistler 9317B 3-component piezoelectric dynamometer is mounted on the $\mathrm{X}-\mathrm{Y}$ stages to measure feed, cross-feed and axial cutting forces. The workpiece is set on the dynamometer.

\subsection{Experimental results and discussion}

In this section, the accuracy of the simulated cutting forces of the proposed model is evaluated. The differences of cutting forces between micro-scale and conventional scale end-milling operations are discussed.

The proposed model has been tested on the experimental data of dozens of micro-end-milling cases, and a very good agreement has been observed between the theoretical and experimental results. The average peak difference of all test cases between computational and experimental cutting forces is around 10\%. The cutting conditions of 12 test

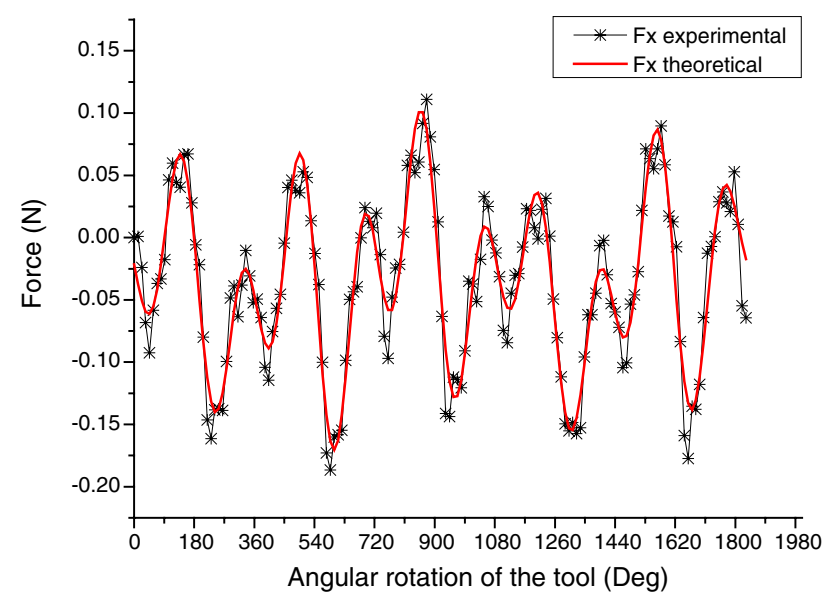

(a)

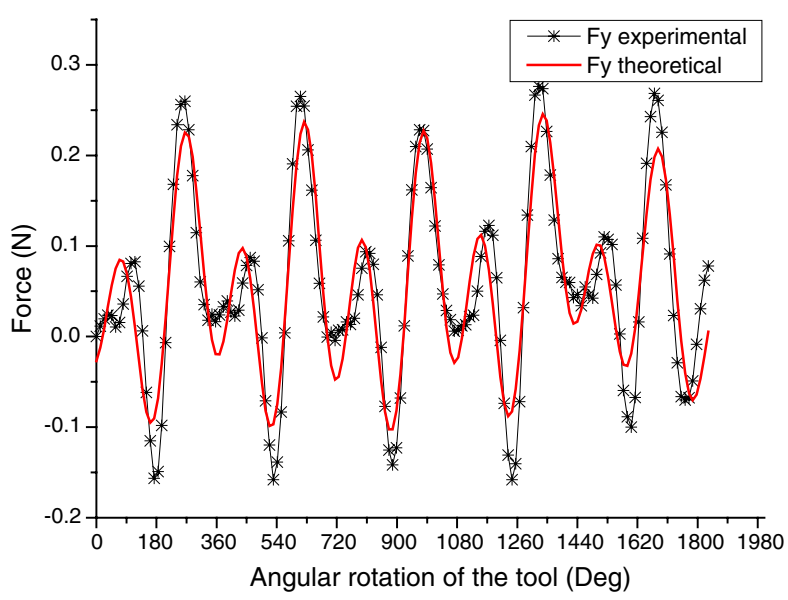

(b)

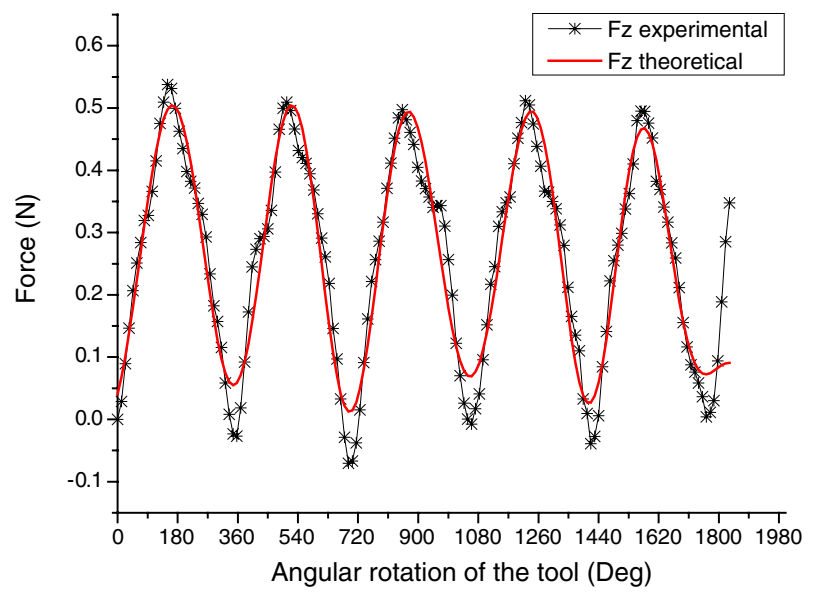

(c)

Figure 9. A comparison between experimental and proposed theoretical cutting forces of slot-milling (case 1: feed rate $0.4 \mathrm{~mm} \mathrm{~s}^{-1}$, axial depth of cut $0.02 \mathrm{~mm}$ ). (a) Feed cutting force $F_{x}$, (b) cross-feed cutting force $F_{y}$ and $(c)$ axial cutting force $F_{z}$.

cases are listed in table 2. First, a two-flute carbide end-mill with $0.8 \mathrm{~mm}$ diameter is slot-milled on a copper workpiece with different operating conditions (from case 1 to 9). The collected cutting force data of nine operating conditions were 


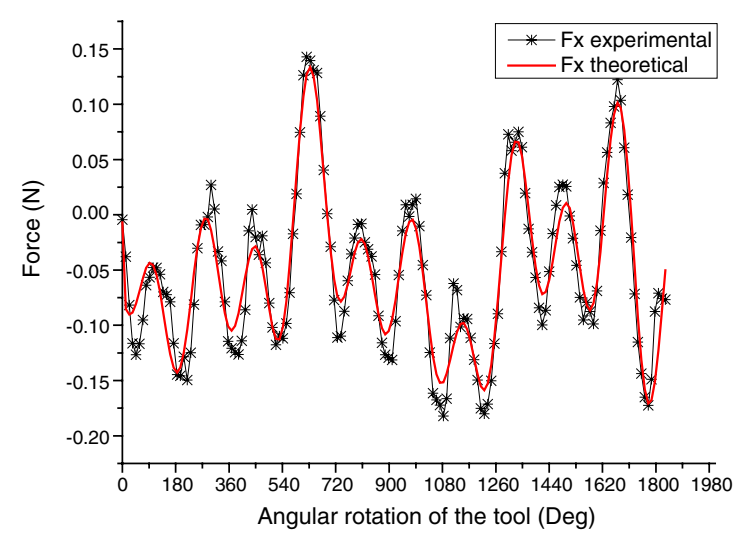

(a)

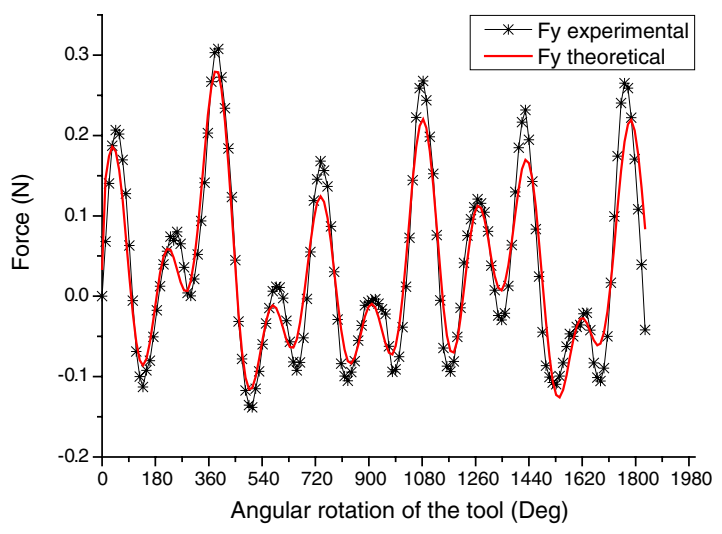

(b)

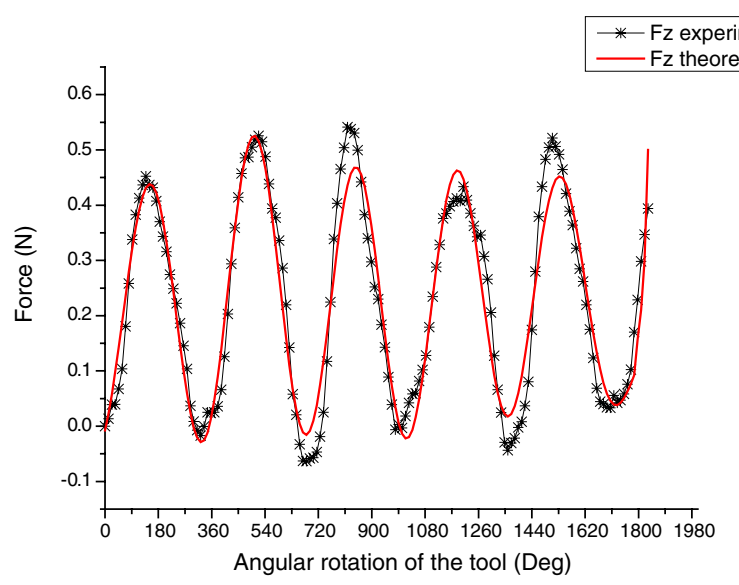

(c)

Figure 10. A comparison between experimental and proposed theoretical cutting forces of up-milling (case 10: feed rate $0.4 \mathrm{~mm} \mathrm{~s}^{-1}$, axial depth of cut $0.02 \mathrm{~mm}$, radial depth of cut $0.4 \mathrm{~mm}$ ). (a) Feed cutting force $F_{x},(b)$ cross-feed cutting force $F_{y}$ and (c) axial cutting force $F_{z}$.

used to decide the cutting force coefficients of the proposed model. The minimum chip thickness was identified as $0.9 \mu \mathrm{m}$ using the method proposed by Kim et al (2004). The other experiments with different feed rates, axial depths of cut and milling methods were performed to verify the proposed model.

The comparisons of two test cases (case 1 and 10) between the simulated and experimental cutting force profiles are

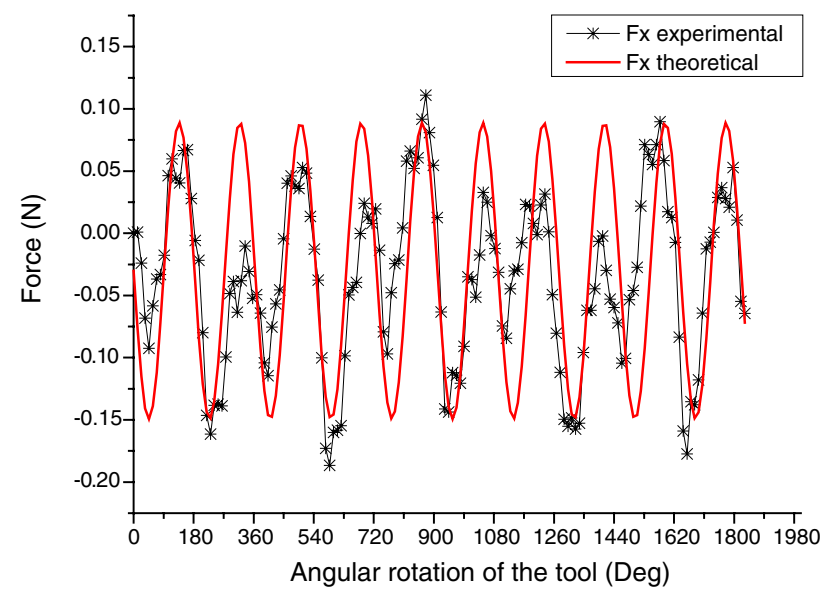

(a)

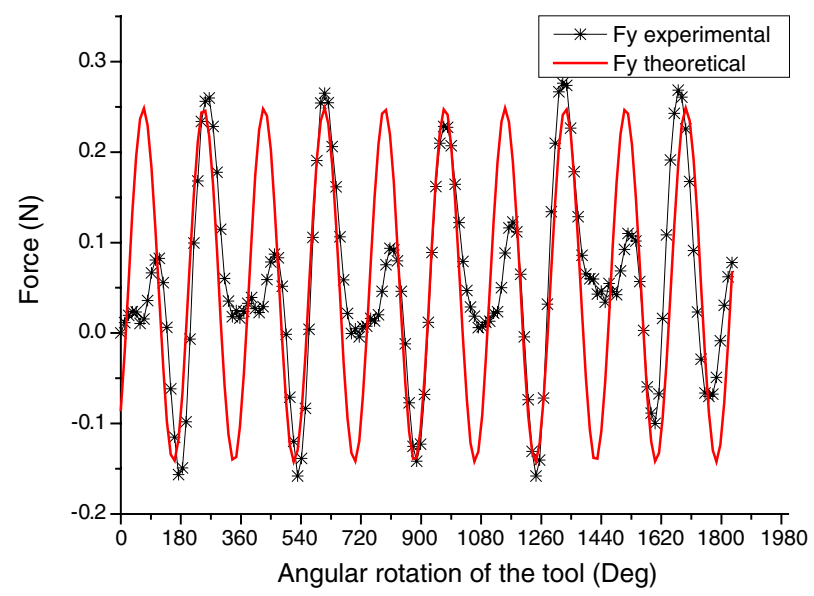

(b)

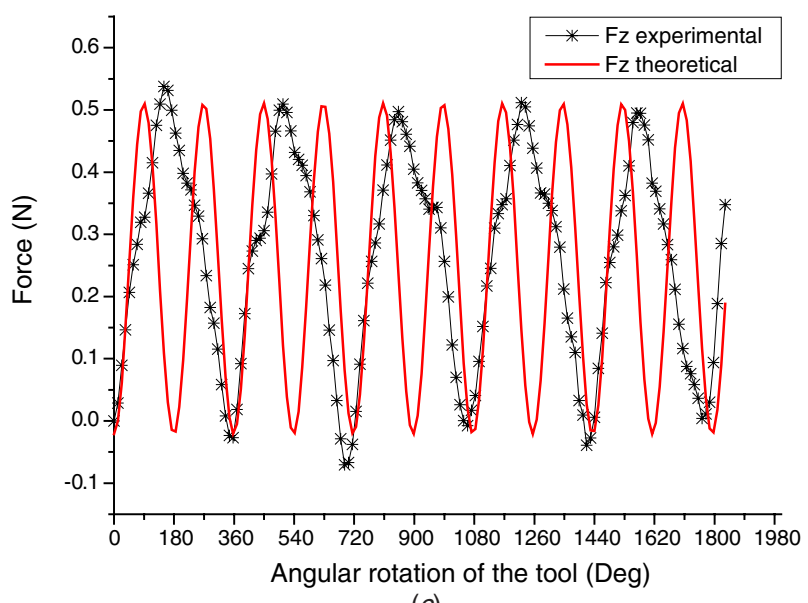

(c)

Figure 11. A comparison between experimental and conventional theoretical cutting forces of slot-milling (case 1: feed rate $0.4 \mathrm{~mm} \mathrm{~s}^{-1}$, axial depth of cut $0.02 \mathrm{~mm}$ ). (a) Feed cutting force $F_{x}$, (b) cross-feed cutting force $F_{y}$ and $(c)$ axial cutting force $F_{z}$.

presented in figures 9 and 10 . The difference between the estimated and experimental maximum cutting forces of the two presented cases was less than $7 \%$.

Due to the neglect of the minimum chip thickness in conventional cutting force models for end-milling, estimated 
Table 2. Cutting conditions.

\begin{tabular}{|c|c|c|c|c|}
\hline \multirow[b]{2}{*}{ Test no. } & \multirow{2}{*}{$\begin{array}{l}\text { Tool diameter: } 0.8 \mathrm{~mm} \\
\text { Spindle speed: } 96000 \mathrm{rpm} \\
\text { Milling method }\end{array}$} & \multicolumn{2}{|c|}{$\begin{array}{l}\text { Workpiece material: } \\
\text { Copper run-out: } 1 \mu \mathrm{m}\end{array}$} & \multirow{2}{*}{$\begin{array}{l}\text { Helix angle } \beta: 30^{\circ} \\
\text { Two-flute cutter } \\
\text { Radial depth } \\
\text { of cut (mm) }\end{array}$} \\
\hline & & $\begin{array}{l}\text { Feed rate } \\
\left(\mathrm{mm} \mathrm{s}^{-1}\right)\end{array}$ & $\begin{array}{l}\text { Axial depth } \\
\text { of cut (mm) }\end{array}$ & \\
\hline 1 & Slot-milling & 0.4 & 0.02 & Full cut \\
\hline 2 & Slot-milling & 0.5 & 0.02 & Full cut \\
\hline 3 & Slot-milling & 0.6 & 0.02 & Full cut \\
\hline 4 & Slot-milling & 0.4 & 0.04 & Full cut \\
\hline 5 & Slot-milling & 0.5 & 0.04 & Full cut \\
\hline 6 & Slot-milling & 0.6 & 0.04 & Full cut \\
\hline 7 & Slot-milling & 0.4 & 0.06 & Full cut \\
\hline 8 & Slot-milling & 0.5 & 0.06 & Full cut \\
\hline 9 & Slot-milling & 0.6 & 0.06 & Full cut \\
\hline 10 & Up-milling & 0.4 & 0.02 & 0.4 \\
\hline 11 & Up-milling & 0.6 & 0.02 & 0.4 \\
\hline 12 & Up-milling & 0.6 & 0.04 & 0.4 \\
\hline
\end{tabular}

cutting forces periodically fluctuate with a frequency equal to the product of the spindle frequency and the number of the tool teeth. However, in a micro-scale end-milling operation, practical cutting forces may periodically fluctuate with the number of tooth passes as shown in figures 9 and 10. The presented model can precisely describe the micro-end-milling operation, and the simulation results show a very satisfactory agreement with those data from milling experiments.

In comparison, figure 11 shows the three-dimensional cutting forces of slot-milling determined by a conventional theoretical model and experimental case 1 . It is clear that the conventional theoretical cutting forces periodically fluctuate with the same periodicity, namely $\pi / \omega$, due to ignoring the effects of the spindle run-out and the intermittency of chip formation. This means that the conventional cutting force model for macro-scale milling cannot accurately predict the force characteristics of the micro-scale milling process.

\section{Conclusions}

In this study, an accurate three-dimensional cutting force model for micro-scale end-milling has been developed by considering the combination of an exact trochoidal trajectory of the tool tip, tool run-out and minimum chip thickness effect due to the intermittency of the chip formation at a microscale. The proposed model has been validated by dozens of micro-end-milling experiments. The average peak difference of all test cases between the computational and experimental cutting force was around $10 \%$. From this work, the following conclusions may be drawn.

(1) At the micro-scale, the milling tool may rotate several times without removing any material, especially at low feed per tooth. So cutting forces may periodically fluctuate with the number of tooth passes. The periodicity of cutting forces is a function of the minimum chip thickness and cutting conditions.

(2) In micro-end-milling operations, the chip formation process and cutting forces are significantly influenced by the trochoidal trajectory of the tool tip, tool run-out and minimum chip thickness, and a relatively large tool runout may result in the feed per tooth for each tooth pass to be no longer a constant.
(3) The proposed model can be used to estimate the operating conditions from the monitored cutting force data. Also, tool breakage and wear can be estimated by inspecting the estimated cutting force coefficients.

\section{Acknowledgment}

The authors gratefully acknowledge the financial support of this research program provided by the National Natural Foundation of China under grant no. 50575134.

\section{References}

Bao W Y and Tansel I N 2000a Modeling micro-end-milling operations: I. Analytical cutting force model Int. J. Mach. Tool Manuf. 40 2155-73

Bao W Y and Tansel I N 2000b Modeling micro-end-milling operations: II. Tool run-out Int. J. Mach. Tools Manuf. 40 2175-92

Bao W Y and Tansel I N 2000c Modeling micro-end-milling operations: III. Influence of tool wear Int. J. Mach. Tools Manuf. 40 2193-211

Kim C-J, Mayor J R and Ni J 2004 A static model of chip formation in microscale milling J. Manuf. Sci. Eng. 126 710-8

Kline W A, Devor R E and Shareef I 1982 Prediction of surface accuracy in end milling J. Eng. Ind. 104 272-8

Montgomery D and Altintas Y 1991 Mechanism of cutting force and surface generation in dynamic milling J. Eng. Ind. 113 160-8

Tlusty J and MacNeil P 1975 Dynamics of cutting forces in end milling CIRP Ann. 24 21-5

Vogler M P, Devor R E and Kapoor S G 2003 Microstructure-level force prediction model for micro-milling of multi-phase materials J. Manuf. Sci. Eng. 125 202-9

Vogler M P, Devor R E and Kapoor S G 2004 On the modeling and analysis of machining performance in micro-end-milling: II. Cutting force prediction J. Manuf. Sci. Eng. 126 695-705

Wang J-J J and Zheng C M 2002 An analytical force model with shearing and ploughing mechanisms for end milling Int. J. Mach. Tools Manuf. 42 761-71

Weule H, Huntrup V and Tritschler H 2001 Micro-cutting of steel to meet new requirements in miniaturization CIRP Ann. 50 61-4

Yuan Z J, Zhou M and Dong S 1996 Effect of diamond tool sharpness on minimum cutting thickness and cutting surface integrity in ultraprecision machining J. Mater. Process. Technol. 62 327-30

Zaman M T, Kumar A S, Rahman M and Sreeram S 2006 A three-dimensional analytical cutting force model for micro end milling operation Int. J. Mach. Tools Manuf. 46 353-66 